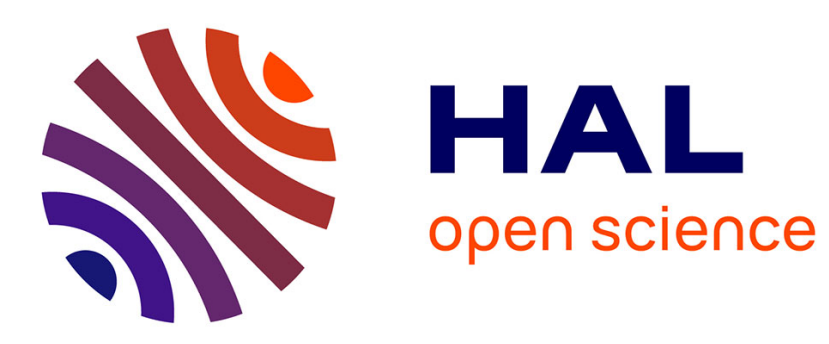

\title{
Stochastic Multiscale Segmentation Constrained by Image Content
}

Luc Gillibert, Dominique Jeulin

\section{To cite this version:}

Luc Gillibert, Dominique Jeulin. Stochastic Multiscale Segmentation Constrained by Image Content. 10th International Symposium on Mathematical Morphology and Its Application to Signal and Image Processing, ISMM 2011, Jul 2011, Verbania-Intra, Italy. pp.132-142, 10.1007/978-3-642-21569-8_12 . hal-00577776

\section{HAL Id: hal-00577776 https://hal.science/hal-00577776}

Submitted on 17 Mar 2011

HAL is a multi-disciplinary open access archive for the deposit and dissemination of scientific research documents, whether they are published or not. The documents may come from teaching and research institutions in France or abroad, or from public or private research centers.
L'archive ouverte pluridisciplinaire HAL, est destinée au dépôt et à la diffusion de documents scientifiques de niveau recherche, publiés ou non, émanant des établissements d'enseignement et de recherche français ou étrangers, des laboratoires publics ou privés. 


\title{
Stochastic Multiscale Segmentation Constrained by Image Content
}

\author{
Luc Gillibert and Dominique Jeulin \\ Centre de Morphologie Mathematique \\ Ecole des Mines de Paris \\ 35, rue Saint Honoré \\ 77305 Fontainebleau, France
}

\begin{abstract}
We introduce a noise-tolerant segmentation algorithm efficient on 3D multiscale granular materials. The approach uses a graphbased version of the stochastic watershed and relies on the morphological granulometry of the image to achieve a content-driven unsupervised segmentation. We present results on both a virtual material and a real X-ray microtomographic image of solid propellant.
\end{abstract}

Keywords: Granular media, granulometry, multiscale 3D segmentation, stochastic watershed, constrained segmentation.

\section{Introduction}

The stochastic watershed segmentation was first introduced by Angulo and Jeulin in [1]. The approach is based on using a large number of realizations of random markers to build a probability density function (pdf) of contours, starting from a standard watershed algorithm producing oversegmentations.

The stochastic watershed was proved to be efficient for unsupervised segmentation [10][6]. The two parameters used for its construction are $k$, the number of random markers used in each realization, and $R$, the number of realizations. From the law of large numbers, the pdf converges when increasing $R$. The parameter $k$ needs to be proportional to the number of desired regions in the segmented image. Therefore, in the case of granular materials, $k$ needs to be proportional to the number of grains contained in the image, that can be automatically estimated. In [6], the authors use the covariance for this estimation, deduced from the average radius of the grains, and a Boolean model assumption [8].

For multiscale images, the covariance is not so efficient. In this paper, we introduce a new approach for stochastic segmentation which relies on the full granulometry of the image. Using morphological openings, this granulometry can be automatically computed from the image and is used as a constraint during iterations of segmentation steps.

\section{Stochastic Segmentation}

\subsection{Stochastic Watershed}

The first method introduced for computing the stochastic watershed is based on a large number of realizations of random markers to estimate a pdf of con- 
tours, or of surface boundaries in 3D. The random markers are generated with an uniform distribution corresponding to a constant intensity. In the case of granular materials, a constant background marker, extracted by an automatic thresholding, is added to each set of random markers. For each set of markers, a constrained watershed is computed. Then, the Parzen window is used to estimate the pdf of contours.

For a good estimation of the stochastic watershed, 100 to 200 realizations are required [1]. However, using $\lambda$-flat zones, a stochastic watershed segmentation can be achieved with only 50 realizations [6]. This number is low, but the computation of 50 watersheds is very time-consuming, especially on large $3 \mathrm{D}$ data sets.

From the pdf, it is possible to obtain the segmentation. The first approach uses this pdf as a gradient for a new watershed [1]. A more efficient approach uses $\lambda$-flat zones to overcome the fact that the estimated pdf is not constant over each branch of contour [6]. Illustration of the pdf of contours is on Fig. 1 (A). The resulting segmentation is illustrated on Fig. 2 (A).

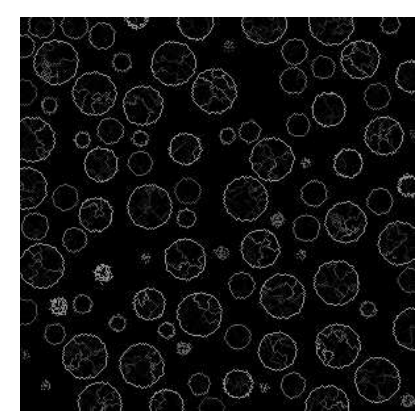

(A)

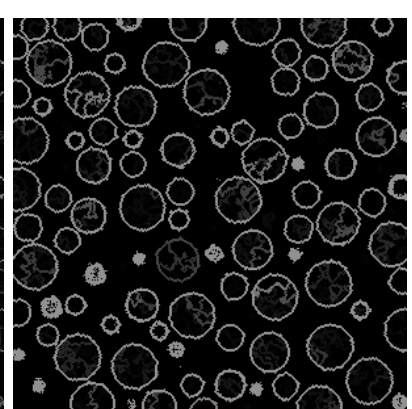

(B)

Fig. 1. (A) Pdf of contours on a simulated $3 D$ material $R_{1}$, estimated with 50 realizations of a Poisson point process (slice). (B) Pdf of contours computed with a graph-based approach (slice).

\subsection{Graph-Based Stochastic Watershed}

Computing a large number of watersheds from simulations provides good results but is a slow process, mainly in $3 D$. A more efficient solution for computing stochastic watersheds is to use a graph-based approach. Probability of boundaries is directly computed with a good approximation without the use of any realisation $[9,7]$.

A first watershed is computed from the local minima of the gradient, as in standard segmentation, but restricted to the complementary set of the background extracted by an automatic thresholding. For this purpose, the background is used as a marker. In the present case, a very strong oversegmentation is obtained as a result of the presence of noise. From this watershed, an adjacency graph is constructed. Vertices of the graph are associated to each basin 


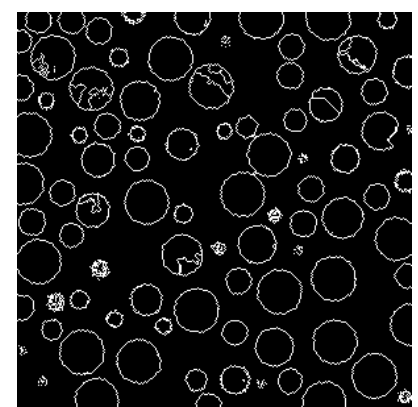

(A)

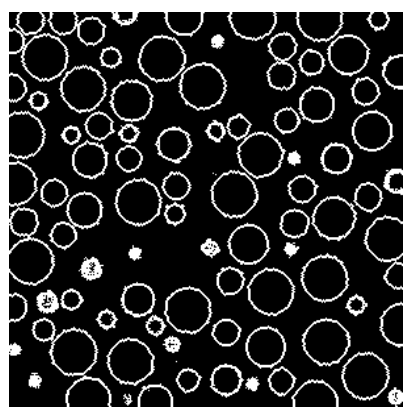

(B)

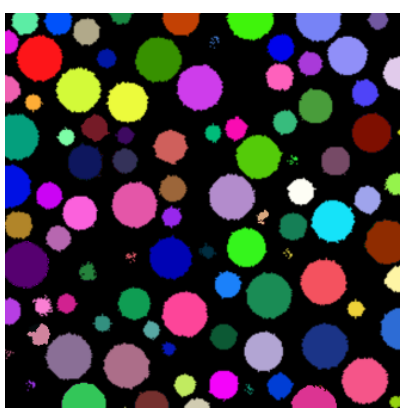

(C)

Fig. 2. (A) Stochastic watershed segmentation (slice). The $\lambda$-flat zones are used. A few grains are over-segmented. Small grains are missing (B) Graph-based stochastic watershed segmentation with a threshold of 0.5 on the probability (slice). A few oversegmented grains, as well as small grains are missing. (C) Final granulometry-driven multiscale segmentation result for the random material $R_{1}$ (slice of a $3 D$ image).

of the watershed, connecting edges between adjacent regions. A vertex is associated to the background too. Values are given to the vertices corresponding to the volumes of the regions (or to the areas, in 2D). Each edge is labelled with the minimum of the gradient function on the boundary between the corresponding regions. From this valued graph, a minimum spanning tree is extracted [7]. Then the regions in the minimum spanning tree are merged, starting with the edge of lowest value. After each merging, the probability $p$ of the boundary between the corresponding regions is estimated using the following equation [9]:

$$
\left.p=1-\left(1-V_{1} / V\right)^{k}-\left(1-V_{2} / V\right)^{k}+\left(1-\left(V_{1}+V_{2}\right) / V\right)\right)^{k}
$$

This is the probability of obtaining at least one random marker in each of the two regions, knowing the volumes $V_{1}$ and $V_{2}$ of the two regions, the total volume $V$ of the image and the number of markers $k$. It is seen from equation 1 that the probability $p$ increases with the volume of the grain, that may cause a bias towards largest grains if there is a wide distribution of sizes in the image.

After the merging of all the nodes in the original minimum spanning tree, the probability of all the edges of the tree is known. The result is projected from the tree on the graph and from the graph on the image.

This approach provides uniform probability on each part of boundary between two regions, as illustrated on Fig. 1 (B). Therefore, the $\lambda$-flat zones are useless and a simple threshold can be used for the segmentation Fig. 2 (B).

\section{Multiscale Image Segmentation}

The multiscale image segmentation process is based on a simple idea: estimate the full granulometry of the image, using morphological openings, then use multiple stochastic watersheds with different numbers of markers for each size, and 
finally combine them to get a segmentation that is pertinent for each size of grains. For achieving this goal, a hierarchy on the boundaries of the stochastic watershed is required. Many hierarchical segmentation algorithms have been studied, as the waterfalls [3] or the P algorithm [4]. Here we introduce an approach similar to the one used for the computation of the waterfall: the merging of the watersheds basins using a minimum spanning tree [5].

\subsection{Hierarchy on Boundaries}

Each boundary is an edge in the adjacency graph. For a non-multiscale graphbased stochastic watershed, a fixed threshold is efficient. So, a first approach for constructing a hierarchy on the edges of the adjacency graph is to use a threshold. All the edges with a probability less than a given value $t$ are removed from the graph. With $t \in[0,1]$, a complete hierarchy is obtained [9].

This approach is fast and easy to compute, but, for a large value of $t$ the remaining edges are not exploitable as boundaries for a segmentation. The removal of edges implies the removal of boundaries and therefore the merging of regions in the images. This process changes the probability of the boundaries of the remaining regions if we iterate the segmentation. This phenomenon is ignored with a fixed threshold. As seen on Fig. 2 (B), some over-segmented grains will disappear by a simple trheshold.

Working with a graph, it is possible to update the probability of the boundaries of the remaining regions at each edge deletion with the following iterative algorithm [9]:

- While the MST has at least 2 vertices.

- In the MST, the edge $e$ with the lowest probability is chosen.

- The edge $e$ is deleted and the adjacent vertices are merged.

- The probability of the edges incident to the merged vertex is updated, using Eq. 1 and the new volume of the vertex.

This leads to better hierarchy on boundaries. As seen on Fig. 3, with the threshold approach some over-segmented grains disappear easily, while this problem is solved with the merging algorithm.

\subsection{Granulometry-Driven Multiscale Approach}

In order to get a pertinent segmentation starting from a highly over-segmented image, we can introduce constraints in the merging process. In the present case, the first step of the approach is to estimate the full granulometry of the image, using morphological openings. For fast computations, we use a rhombicuboctahedron as structuring element and we work on the binarized (thresholded) image. Gaussian noise, border effects and the shape of the structuring element leads to a few errors on the granulometry, as seen on Fig. 4, but they will not induce errors in the segmentation process. 


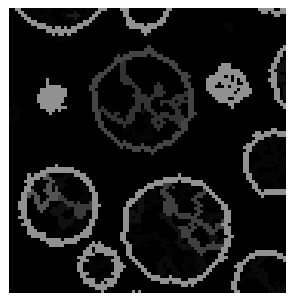

(A)

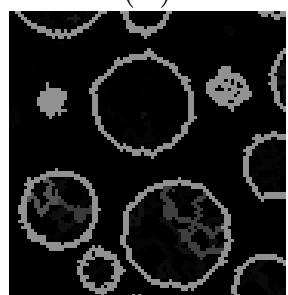

(E)

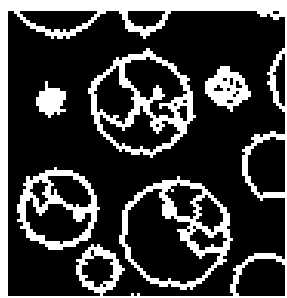

(B)

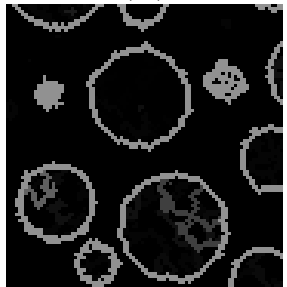

(F)

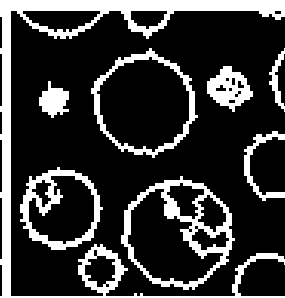

(C)

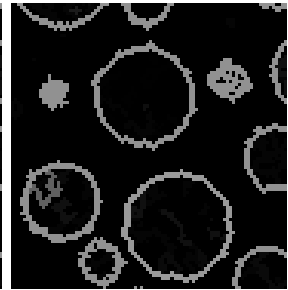

(G)

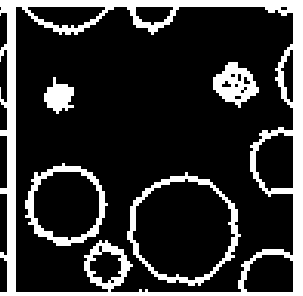

(D)

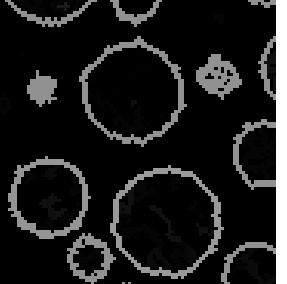

(H)

Fig. 3. (A) Probability density function of contours on a $3 D$ material $R_{1}$, computed with a graph-based approach (detail of a slice). (B), (C), (D): hierarchy on boundaries using increasing thresholds. $(\mathrm{A}),(\mathrm{E}),(\mathrm{F}),(\mathrm{G}),(\mathrm{H})$ : hierarchy on boundaries using the merging algorithm, step 0 is $(\mathrm{A})$.

From the granulometry, a small number of classes are chosen. A good approach for this choice is maximising the interclass variance. For our sample material $R_{1}$, we uses 3 classes: radius [13,18], radius $[6,13]$ and radius $[1,5]$. The total volume of the grains in each class will be denoted $v(x)$. The number of grains in each class is deduced from $v(x)$ and is denoted $n(x)$. It is used to generate the corresponding number of markers in the calculation of the probability.

Then, the standard watershed is computed from the local minima of the gradient. From this watershed, the adjacency graph is constructed and a minimum spanning tree is extracted.

The first class is chosen. The stochastic watershed is computed with a number of markers equals to $n(1)$. In our $3 D$ example (material $R_{1}$ ), 417 markers are needed. Based on this stochastic watershed, a first hierarchy on boundaries is computed with the merging algorithm. For each step $i$ of the hierarchy, the granulometry of the corresponding segmentation is computed $\left(v_{i}(1)\right)$.

In the full hierarchy, there is a size step $i^{*}$ which minimises the difference $\left|v_{i}(1)-v(1)\right|$. In our example, $i^{*}=40$ (Fig. 11 and Fig. 5). This step is used for the segmentation of the grains in the first class. All the segmented grains are removed from the image and added to the background mask. The minimum spanning tree is updated and the next class is chosen.

The same process is applied to all the classes. When no more class are left, all the segmentations are combined together. This provides the final result illustrated on Fig. 2 (C) and Fig. 12. The main lines of the multiscale image segmentation process are summarized on Fig. 6. 


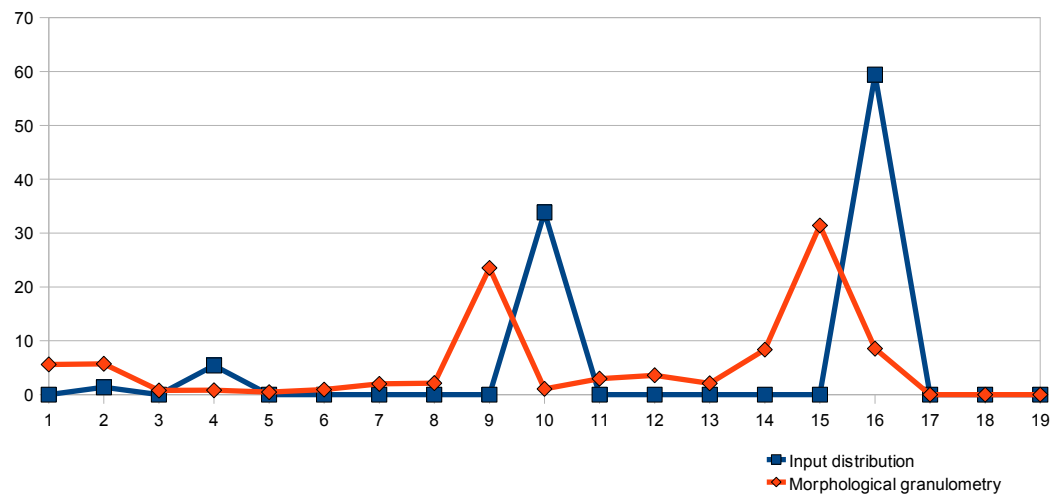

Fig. 4. Input granulometry of the random material $R_{1}$ and granulometry measured on the thresholded image from morphological openings. On $x$ axis, the radius, and on $y$ axis the volume fraction of grains with this radius (in percent).

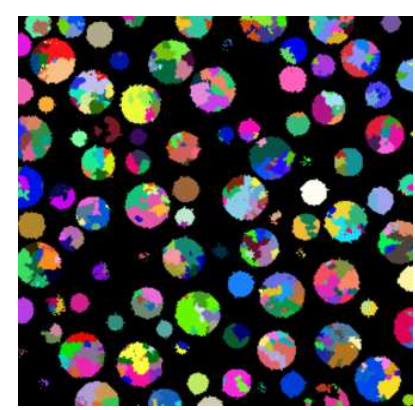

(A)

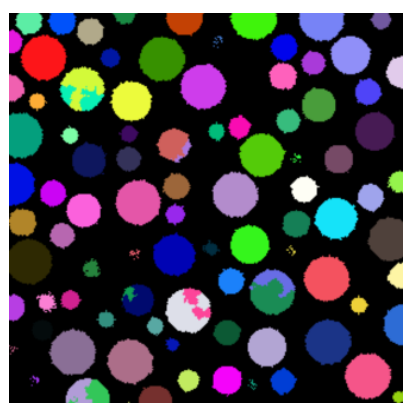

(B)

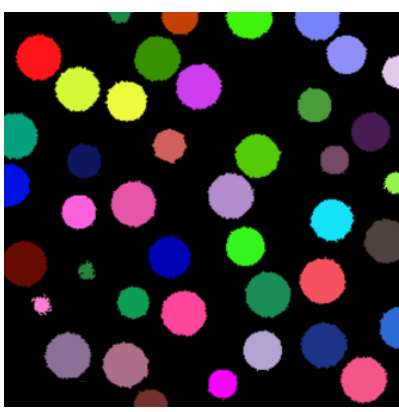

(C)

Fig. 5. The segmentation of the random material $R_{1}$ (slice). Results for the class 1 on steps 1 (A), 20 (B) and 40 (C).

\section{Validation}

\subsection{Random Model}

For the validation, we use a simple random model. We generate 7560 random discrete spheres. The distribution of the radius of the spheres is fixed and is given on Fig. 4. The centres of the spheres are uniformly distributed in a cubic volume of size $300 \times 300 \times 300$ with a rejection sampling algorithm (acceptance-rejection method). Two spheres cannot overlap more than a given threshold (fixed to 2 voxels). Spheres have non-uniform grey values, and show a visible boundary generating information in the gradient image. The volume fraction of the grains on the sample is 0.432 . A strong Gaussian noise is finally added. A slice of the simulated material and of the corresponding binary mask is illustrated on Fig. 7. 


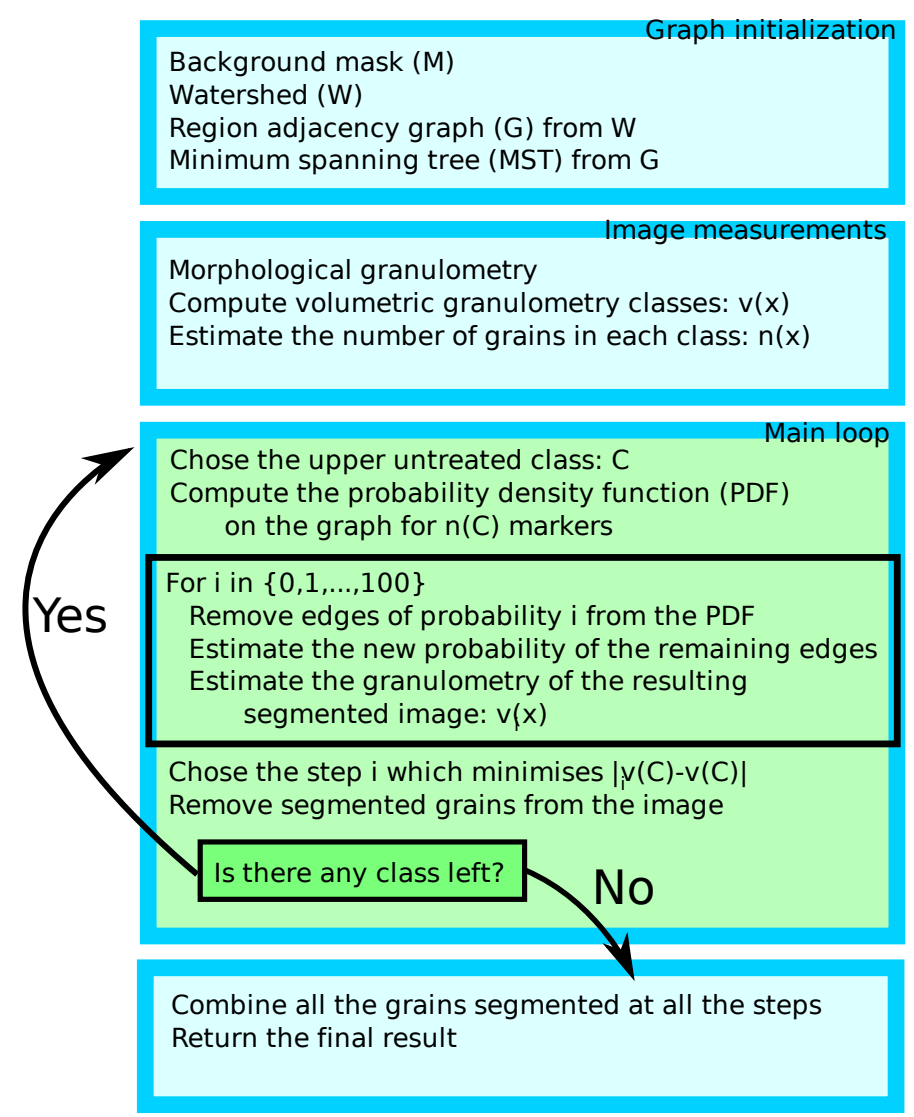

Fig. 6. The main steps of the multiscale image segmentation process.

\subsection{Microtomographic Images}

Our second sample is an X-ray microtomographic image provided by the CEA Gramat. The image was generated at with a Skyscan 1172 high-resolution microCT. We are operating on a $954 \times 243 \times 243$ subimage. It is a solid propellant sample with fragmented grains due to a mechanical impact. A voxel is $1.80 \mu \mathrm{m}$. The original diameter of the grains is $400 \mu \mathrm{m}$, but there are many small fragments. A slice is shown on Fig. 8. Results of the segmentation are illustrated on Fig. 10 and Fig. 9, where it is clear that the multiscale approach overcomes the standard stochastic watershed algorithm.

\section{Conclusion}

On both the simulated image and the X-ray microtomographic image, our multiscale approach provides a good segmentation (it is essential to use a "volume" weighted granulometry, all our attempts with a "number" granulometry being 


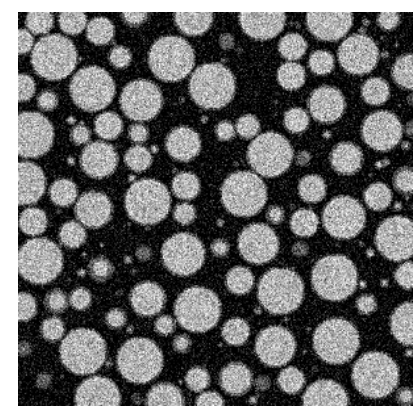

(A)

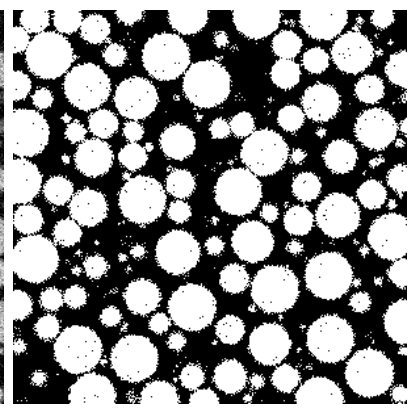

(B)

Fig. 7. (A) Random material $R_{1}$ with a strong Gaussian noise and a wide grain size distribution (slice). (B) The binary mask for material $R_{1}$ (slice), the threshold is calculated via the maximization of the interclass variance.

\begin{tabular}{|r|r|r|}
\hline Algorithm & Image & Time \\
\hline \hline Stochastic Watershed & Random material $R_{1}$ & $1 \mathrm{~h} 36 \mathrm{~m} 36 \mathrm{~s}$ \\
& Propellant (microtomographic) & $3 \mathrm{~h} 9 \mathrm{~m} 21 \mathrm{~s}$ \\
\hline Graph-Based Stochastic Watershed & Random material $R_{1}$ & $5 \mathrm{~m} 11 \mathrm{~s}$ \\
& Propellant (microtomographic) & $14 \mathrm{~m} \mathrm{9s}$ \\
\hline Multiscale Image Segmentation & Random material $R_{1}$ & $9 \mathrm{~m} 22 \mathrm{~s}$ \\
& Propellant (microtomographic) & $25 \mathrm{~m} \mathrm{7s}$ \\
\hline
\end{tabular}

Table 1. Computational cost of the stochastic watershed, the graph-based stochastic watershed and our multiscale image segmentation approach. Times are give on a 3.00 GHz Pentium 4.

unsuccessful, due to its inherent sensitivity to noisy data). On the simulation, there is no over-segmentation for large grains, and the small grains are present as illustrated on Fig. 2 (C). On the microtomographic image, some of the large grains are over-segmented, but most of the small fragments are present and the results are better than the original stochastic watershed approach as illustrated on Fig. 10.

The granulometry of the segmented image fits to the morphological granulometry of the input images, since it was used as a constraint in the merging process (Fig. 12 and Fig. 9).

This new segmentation technique, combining an iterative probability-based merging of boundaries and a size distribution constraint, is very robust with respect to the noise contained in the image, without the necessity to apply a filter that would destroy the smallest grains. In addition, the segmentation is fully non-parametric, since at every step the required parameters are automatically deduced from the image.

Due to the graph approach, the process is much faster than the original stochastic watershed, as summarized on Tab. 1.

Finally, it is possible to generalize the process to multi-criteria segmentation, using any other measurement tool as a constraint during the multiscale segmentation. 


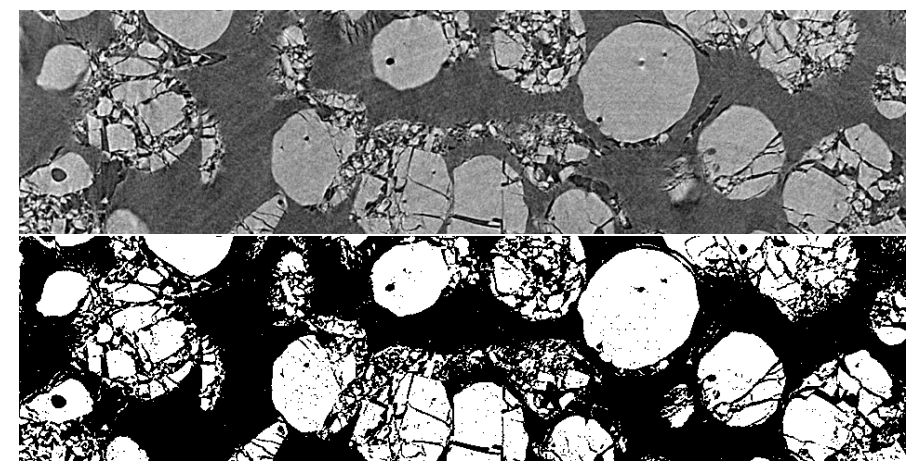

Fig. 8. Image $E_{1}$ : a $3 \mathrm{D}$ X-ray microtomographic image of a fragmented granular material and its binarization by maximisation of the interclass variance (slice).

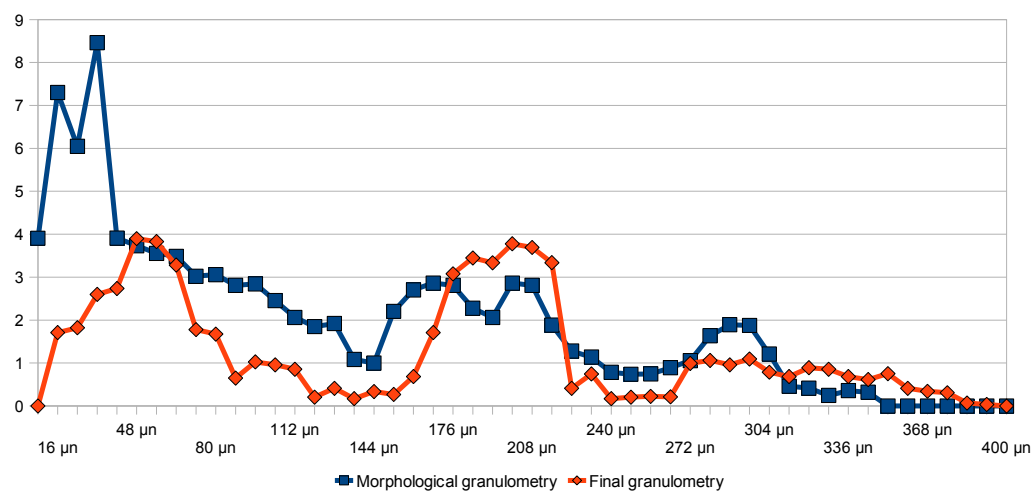

Fig. 9. Morphological granulometry of the X-ray microtomographic image of a fragmented granular material and granulometry of the final segmented image. On $x$ axis, the diameter $(\mu \mathrm{m})$, and on $y$ axis the volume fraction of grains with this diameter.

Acknowledgments: This work was supported by a grant fom DGA (contract 200934 0006). The authors are grateful to Alain Fanget (CEA Gramat) for his advice during this study.

\section{References}

1. Angulo, J., Jeulin, D.: Stochastic watershed segmentation. Proceedings of ISMM, 8th International Symposium on Mathematical Morphology, ISBN 978-85-17-000324, pages 265-276, 2007.

2. Beucher, S., Lantujoul, C.: Use of watersheds in contour detection. In International workshop on image processing, real-time edge and motion detection (1979).

3. Beucher, S.: Watershed, hierarchical segmentation and waterfall algorithm. Proc. Mathematical Morphology and its Applications to Image Processing, Fontainebleau, Kluwer Ac. Publ., Nld, 1994, pages 69-76, Sept. 1994.

4. Beucher, S., Marcotegui, B.: P algorithm, a dramatic enhancement of the waterfall transformation. CMM/Mines Paristech publication, 86 pages. September 2009. 


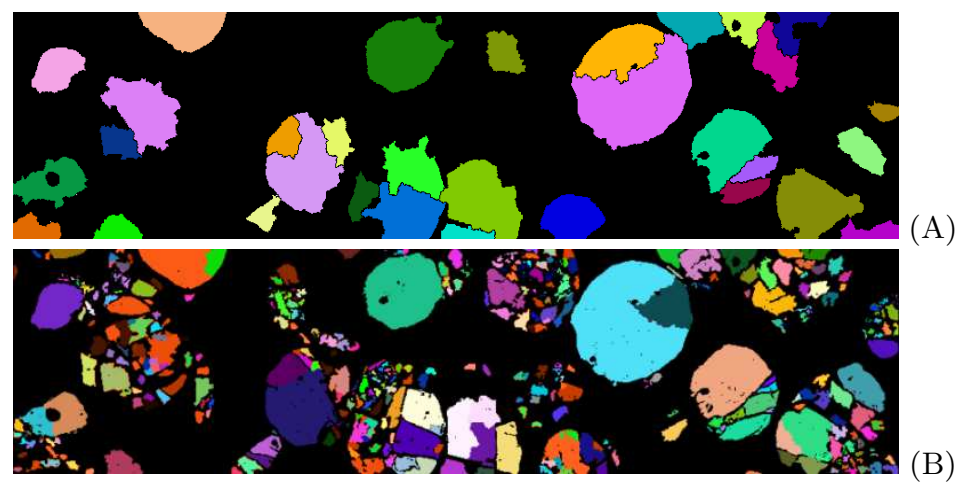

Fig. 10. Segmentation of the X-ray microtomographic image of a fragmented granular material (slice). (A) Results for the stochastic watershed approach. (B) Results for the granulometry-driven multiscale approach.

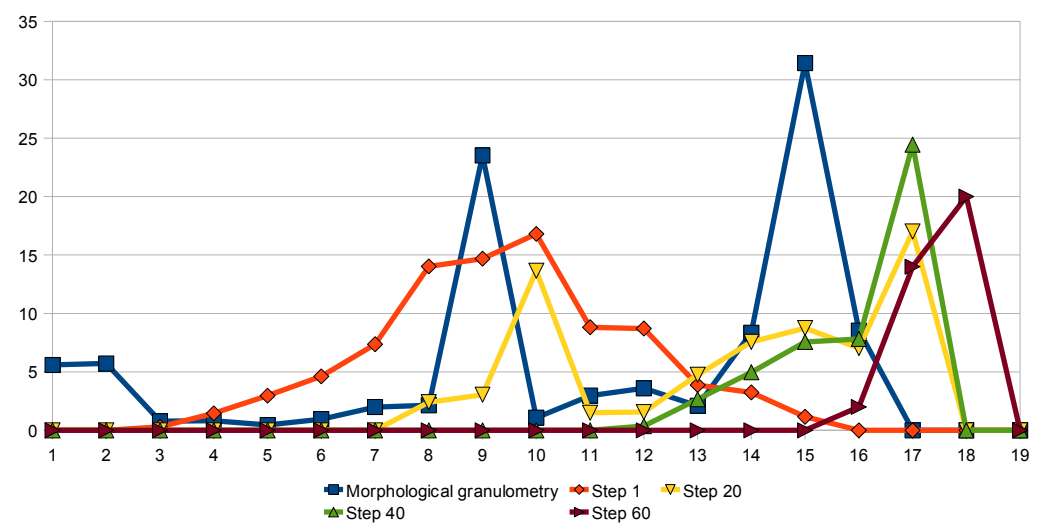

Fig. 11. Morphological granulometry of the random material $R_{1}$ and granulometry obtained at different steps of the hierarchy. The best result is step 40 .

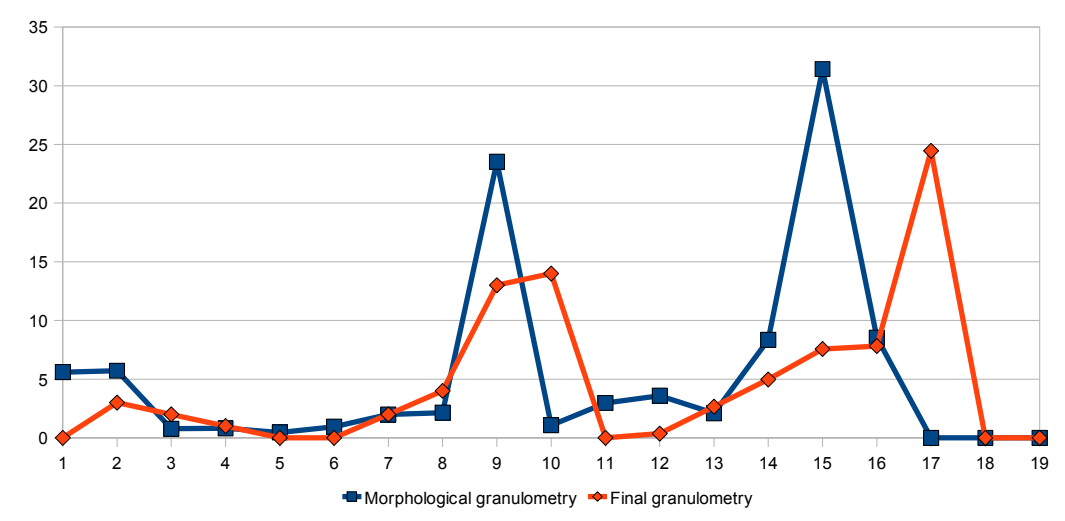

Fig. 12. Morphological granulometry of the random material $R_{1}$ and granulometry of the final segmented image. 
5. Marcotegui, B., Beucher, S.: Fast implementation of waterfall based on graphs. in Mathematical Morphology: 40 Years on : Proc. 7th ISMM. Paris. Springer, pages 177-186, April 2005.

6. Faessel, M., Jeulin, D.: Segmentation of 3D microtomographic images of granular materials with the stochastic watershed. Journal of Microscopy, Volume 239, Issue 1, pages 17-31, July 2010.

7. Meyer, F., Stawiaski, J.: Tailored hierarchies for segmentation, submitted.

8. Jeulin, D.: Modèles morphologiques de structures aléatoires et de changement d'échelle. Thèse de Doctorat d'État, University of Caen, France, 1991.

9. Jeulin D.: Remarques sur la segmentation probabiliste, N-10/08/MM, Internal Report, Mines ParisTech, September 2008.

10. Noyel, G., Angulo, J., Jeulin, D.: Random germs and stochastic watershed for unsupervised multispectral image segmentation. Proceedings of KES 2007. 